\title{
Nanoscale domain wall engineered spin-triplet Josephson junctions and SQUID
}

\author{
Ekta Bhatia, ${ }^{\dagger}$ Anand Srivastava, ${ }^{\ddagger}$ James Devine-Stoneman, ${ }^{\ddagger}$ Nadia A. \\ Stelmashenko, ${ }^{\ddagger}$ Zoe H. Barber, $\stackrel{\ddagger}{\ddagger}$ Jason W. A. Robinson,$\stackrel{\ddagger}{\ddagger}$ and Kartik Senapati ${ }^{* \dagger}$ \\ $\dagger$ School of Physical Sciences, National Institute of Science Education and Research \\ (NISER), HBNI, Bhubaneswar, Odisha, India-752050 \\ $\ddagger$ Department of Materials Science and Metallurgy, University of Cambridge, 27 Charles \\ Babbage Road, CB3 OFS, UK \\ E-mail: kartik@niser.ac.in
}

\section{Experimental details}

Film Growth: Thin film bilayers of $\mathrm{Ni}(110 \mathrm{~nm}) / \mathrm{Nb}(65 \mathrm{~nm})$ stack were prepared at room temperature in an Ar pressure of 1.5 Pa, by dc-magnetron sputtering using high purity $\mathrm{Nb}$ and $\mathrm{Ni}$ targets on $\mathrm{Si} / \mathrm{SiO}_{2}$ substrates. The base pressure of the deposition system was of the order of $10^{-9} \mathrm{mBar}$. Film thicknesses were calibrated by growing films onto patterned substrates and by measuring the height of step edges with an atomic force microscope.

Device Fabrication: Planar Josephson junctions were fabricated by patterning $\mathrm{Ni} / \mathrm{Nb}$ bilayers in the form of stripes using optical lithography and Ar-ion-milling in the first step. Then, Ga-ion Focused Ion Beam (FIB) milling process was used to define the junction by 
making triangular notches and carving a gap of width $\sim 70 \mathrm{~nm}$ in the top Nb layer over the notch area as shown in Fig. 1(a). The dimensions of the gap in the Nb layer were $(70 \times 100) \mathrm{nm}^{2}$. Since a clear separation between the two superconducting electrodes is a key requirement for the success of the experiment, we have made additional FIB cuts in the possible re-deposition areas around the junction to rule out any indirect path for the current flow. Similarly, the side walls of the junction area were also polished with low Ga ion beam current (11 pA) to remove any possible $\mathrm{Nb}$ re-deposition. With these precautions it is very unlikely to have a stray current path across the junctions. ame protocol was followed for the SQUID devices as well.

Micro-magnetic simulations: In order to look at the domain structures for an inplane and out of plane magnetic fields, we have performed 3D object oriented micromagnetic framework (3D OOMMF) simulations using the NIST mif code(Donahue, M. J. OOMMF User's Guide, Version 1.0. National Institute of Standards and Technology: Gaithersburg, MD 20899-8910, 1999). In the simulations, the values of exchange constant and saturation magnetization were taken from the literature (Boardman, P. R. Computer simulation studies of magnetic nanostructures. Ph.D. thesis, University of Southampton, 2006). The x, y and $\mathrm{z}$ dimensions of the samples were kept fixed as $3 \mu \mathrm{m}, 3 \mu \mathrm{m}$ and $90 \mathrm{~nm}$, respectively. Here, $\mathrm{x}$ axis refers to the direction along the length of the stripes, y axis refers to the direction along the width of the stripes and $\mathrm{z}$ axis refers to the axis perpendicular to the sample plane. The cell size for simulation was kept as $(10,10,10) n m^{3}$ in $(\mathrm{x}, \mathrm{y}, \mathrm{z})$ directions.

Similar to the case of a single notch in Ni stripe, we have performed 3D OOMMF simulations for the two notches in a Ni loop. Fig. 3(c) shows the OOMMF simulation image of $\mathrm{Ni}$ loop at remanence state in out of plane field configuration. The $\mathrm{x}, \mathrm{y}$ and $\mathrm{z}$ dimensions of the samples were kept fixed as $6 \mu \mathrm{m}, 3 \mu \mathrm{m}$ and $90 \mathrm{~nm}$, respectively with cell size of $(10,10$, 10) $n m^{3}$ in $(\mathrm{x}, \mathrm{y}, \mathrm{z})$ directions.

Transport measurements: Magnetization $(M)$ measurements were made using a Quantum Design Squid magnetometer with magnetic fields $\left(\mu_{0} H\right)$ applied in the out-of- 
plane direction. Junction measurements were performed in a cryogen-free pump probe system. Current-voltage $I(V)$ characteristic were measured in a four probe current-biased configuration.

\section{Effect of magnetoresistance on the SQUID oscilla- tions of the Nb-Ni-Nb planar SQUID device}

Since the Josephson junctions and the SQUID devices were fabricated from Nb-Ni bilayer tracks, one needs to look at the effect of magnetoresistance of the underlying Ni layer and the junction area on the SQUID oscillation. In the supplementary Figure 1 we plot the SQUID oscillations $(2 \mathrm{~K})$ and the low field magnetoresistance of the device measured at $10 \mathrm{~K}$, just above transition temperature. There is no change in the oscillation period or amplitude of the SQUID oscillations. This shows that the conditions of triplet generation is not affected the magnetoresistance of the Ni layer in the junction area. Only the magnetoresistance background is added to the oscillations causing an increase in the average slope of the data.

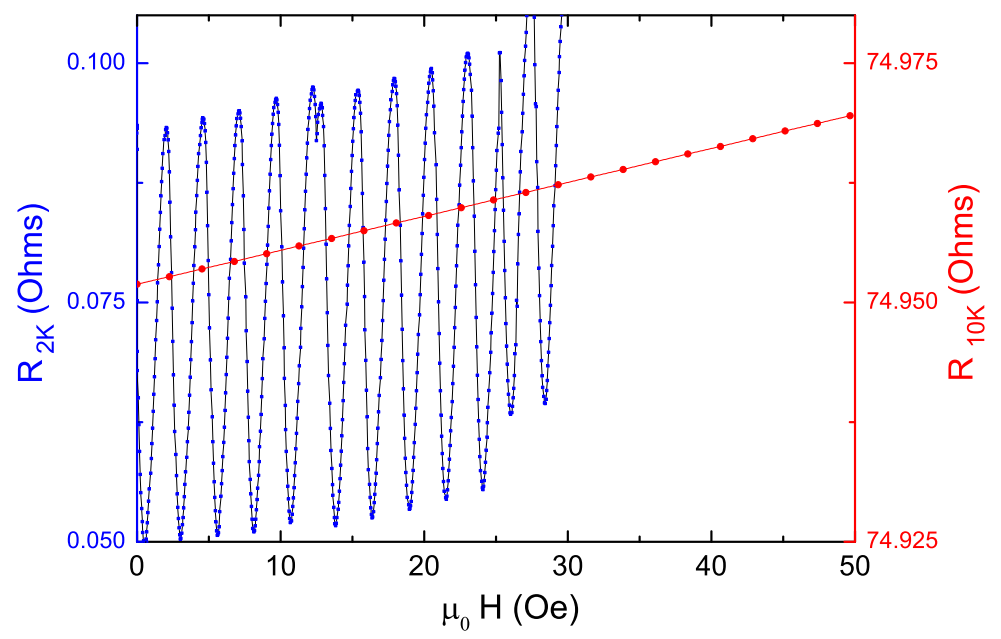

Figure 1: Effect of magnetoresistance on the SQUID oscillations of the Nb-Ni-Nb planar SQUID device : Left hand axis shows the Resistance vs Flux data of the SQUID device at $2 \mathrm{~K}$ and the right hand axis shows the normal state magnetoresistance of the device measured at $10 \mathrm{~K}$. 


\section{Temperature dependence of critical current of a pla- nar Nb-Ni-Nb Josephson device :}

In a planar SNS junction, fabricated from a bilayer geometry, the superconducting electrodes are in contact with the non-superconducting layer underneath, throughout its length. Because of this parallel geometry, some current inevitably shunts through the normal layer $(\mathrm{Ni}$ in this case), causing a normal resistance even in the superconducting state of the electrodes. This explains the finite slope of the IV curves at zero voltage. SNS junctions are strongly overdamped because they are intrinsically shunted by the normal barrier. Therefore, transition from supercurrent to voltage state is usually rounded by thermal noise as reported earlier (Ambegaokar and Halperin, Phys. Rev. Lett. 22, 1364, (1969)). In our case, the magnetic

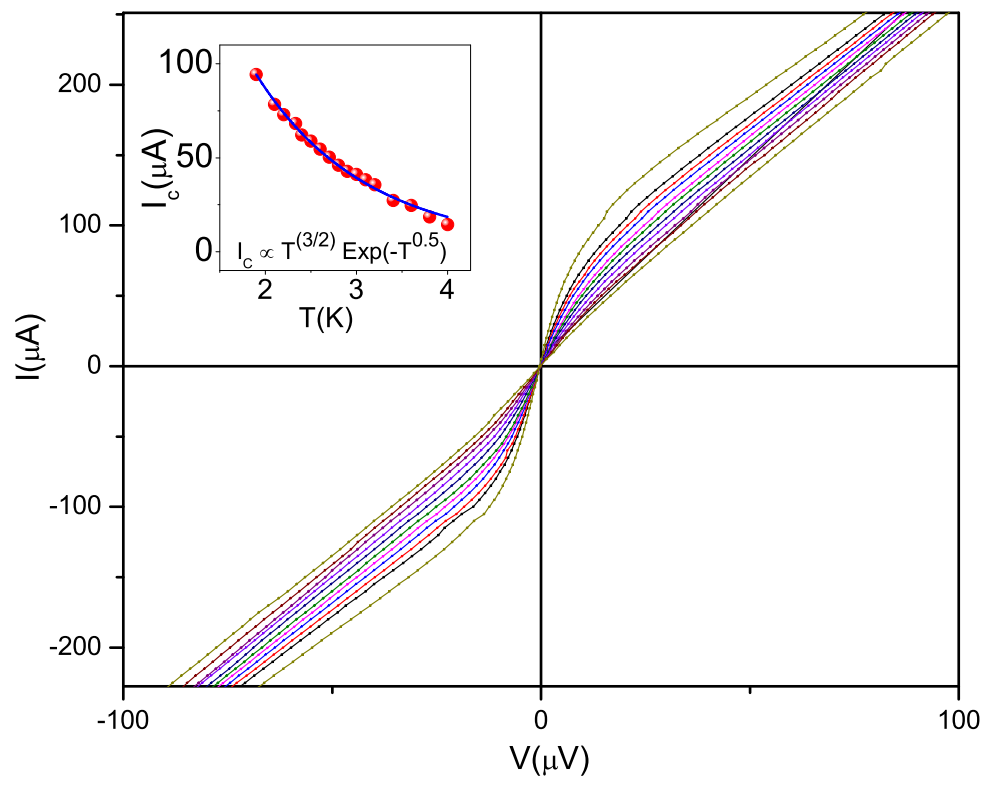

Figure 2: Temperature dependence of critical current of a planar $\mathrm{Nb}-\mathrm{Ni}-\mathrm{Nb}$ Josephson device : The main panel shows the measured I-V curves at several temperatures in the range of $\sim 2 \mathrm{~K}$ to $4 \mathrm{~K}$. The inset shows the critical current as a function of temperature. The solid line is a fit to the dependence $I_{C} \propto T^{\frac{3}{2}} \exp \left(-T^{0.5}\right)$ expected for a long SNS junction.

nature of the barrier further adds to the rounding of the transition by magnetic scattering of the quasiparticles. Therefore, the expected sharp jump in IV curve from superconducting to a finite voltage state is rounded in these junctions. We have observed this effect consistently 
in all Josephson junctions and SQUID devices studied in this work. In spite of the rounding of the IV curves the temperature dependence of critical current (defined at $\pm 5 \mu \mathrm{V}$ ) fits very well to the expected (Dubos et al., Phys Rev B 63, 064502 (2001)) SNS junction behaviour $\left(I_{C} \propto T^{\frac{3}{2}} \exp \left(-T^{0.5}\right)\right)$ for long junctions, as shown in the inset of the supplementary Figure 2. Therefore, the Josephson coupling in these junctions is verified. 